\title{
BMJ Open A nationwide survey on the expectation of public healthcare providers on family medicine specialists in Malaysia - a qualitative analysis of 623 written comments
}

\author{
Boon-How Chew, ${ }^{1}$ Ai-Theng Cheong, ${ }^{1}$ Mastura Ismail, ${ }^{2}$ Zuhra Hamzah, ${ }^{3}$ \\ Mohd-Radzniwan A-Rashid, ${ }^{3}$ Mazapuspavina Md-Yasin, ${ }^{4}$ Norsiah $\mathrm{Ali}^{5}$
}

To cite: Chew B-H,

Cheong A-T, Ismail M, et al. A nationwide survey on the expectation of public healthcare providers on family medicine specialists in Malaysia-a qualitative analysis of 623 written comments. BMJ Open 2014;4:e004645. doi:10.1136/bmjopen-2013004645

- Prepublication history for this paper is available online. To view these files please visit the journal online (http://dx.doi.org/10.1136/ bmjopen-2013-004645).

Received 9 December 2013 Revised 8 May 2014 Accepted 27 May 2014

CrossMark

For numbered affiliations see end of article.

Correspondence to Dr Boon-How Chew; chewboonhow@gmail.com

\section{ABSTRACT}

Objective: To examine the expectation of public healthcare providers/professionals (PHCPs) who are working closely with family medicine specialists (FMSs) at public health clinics.

Design: Cross-sectional study.

Setting: This study is part of a larger national study on the perception of the Malaysian public healthcare professionals on FMSs.

Participants: PHCPs from three categories of health facilities, namely hospitals, health clinics and health offices. Main outcome measures: Qualitative analysis of written comments of respondents' expectation of FMSs.

Results: The participants' response rate was 58\% (780/ 1345 ) with an almost equal proportion from each public healthcare facility. We identified 21 subthemes for the 623 expectation comments. The six emerging themes are (1) need for more FMSs, (2) clinical roles and functions of FMSs, (3) administrative roles of FMSs, (4) contribution to community and public health, (5) attributes improvement and (6) research and audits. FMSs were expected to give attention to clinical duty. Delivering this responsibility with competence included having the latest medical knowledge in their own and others' medical disciplines, practising evidence-based medicine in prehospital and posthospital care, better supervision of staff and doctors under their care, fostering effective teamwork, communicating more often with hospital specialists and making appropriate referral. Expectations ranged from definite and strong for more FMSs at the health clinics to low expectation for FMSs' involvement in research; to mal-expectation on FMSs' involvement in community and public health programmes.

Conclusions: There were some remarkable differences in expectations on FMSs from the three different PHCPs. These ranged from being clinically competent and administratively available for patients and staff at the health clinics, to mal-expectations on FMSs to engage in public health affairs. Relevant parties, including FMSs themselves, could take appropriate self-improvement initiatives to enhance public practice of family medicine and patient care.

Trial registration number: NMRR ID: 08-12-1167.

\section{Strengths and limitations of this study}

The strengths of this study are the nationwide scale of coverage with an almost equal participation of public healthcare providers/professionals from the three different public healthcare facilities.

- Owing to the small sample size at the state level, and the highly unequal contribution from the three different healthcare facilities, the representativeness and generalisability of the findings to any of the states should be viewed cautiously.

- Future work is needed to verify the relevance, practicality and prioritisation of certain clinical roles and functions of family medicine specialists for overall improvement.

\section{INTRODUCTION}

Working together among healthcare professionals on behalf of patients requires teamwork across a complex set of interprofessional relationships and services. ${ }^{1}$ It requires skilful management and a mindset of vigilance that involves a continuous process of improvement. ${ }^{1}$ Such clinical cooperation and collaboration between family physicians and other specialists, either explicit or implicit, are of critical importance to the care of many patients. ${ }^{24}$ This care coordination and cooperation among clinicians have been the priority areas for quality improvement in many clinical practices. ${ }^{5} 6$ Thus, meeting the expectation of other healthcare professionals/providers who work with family medicine specialists (FMSs) could affect the effectiveness of primary healthcare delivery in daily practice. ${ }^{7-9}$

The primary care practice in this country is provided for by two main sectors. ${ }^{10}$ The more prevalent private general practitioners (GPs) are located mostly in and around cities, and service is $100 \%$ paid out of pocket by the patients or through personal health 
insurance schemes and coverage. ${ }^{10}$ GP practices are usually solo practices, and some are in group practices, mostly by non-specialist doctors. On the other hand, public health clinics are quite evenly distributed throughout the country, with smaller clinics in the more remote areas. ${ }^{10}$ The bigger public health clinics have resident doctors and FMSs, and are equipped with complete in-house facilities ranging from medical laboratory tests to plain X-rays to pharmacy. Thus, the public primary care service usually comprises a multidisciplinary team approach in patient care consisting of nutritionists or dieticians, pharmacists, physiotherapists, occupational therapists and paramedics who have undergone specialised training to provide diabetes education, obstetric ultrasonography, eye care, emergency care and others. $^{10}$

The issue of FMSs to be the head of the public health clinics in Malaysia has been controversial and unsettling. The contention and difficulty arise from having to change existing senior medical officers as the head of the clinics with its modus operandi versus newly graduated well-trained FMSs with its transformed approaches and new modes of operation. The other reason is the tension to balance between the clinical and administrative duties of a clinic head that could be overwhelming in a busy public health clinic. The heads of clinics are responsible for the quality of primary medical care, managing clinic staff and are answerable to the local district health offices and directors for issues pertaining to how the clinics are being run under their care. These clinic-administrative duties are mainly around issues involved in direct patient care on a day-to-day basis. Health planning, clinics structure maintenance, performance reviews, annual budgeting, staff remuneration and final assessment of all categories of staff at the clinic would be coordinated and managed by the district health directors (usually public health physicians by training) at district health offices. ${ }^{10}{ }^{11}$ Any perceived major administrative issues at the clinics by the clinic heads would also be referred to district health offices that have the support of the state health offices. Other members helping in the administrative jobs at the district health offices consist of nursing managers, the inspectorate and administrative staffs. ${ }^{11}$

Public health clinics are linked to each other and to public hospitals with an established referral system that is as seamless as a referral can be within a healthcare facility. ${ }^{10}{ }^{11}$ Through this referral system, patients could enter into the public health system at any healthcare facility and be referred to any other healthcare facilities for further medical or surgical care including by the clinical specialists at hospitals. These public hospitals comprise the more adjacent and smaller public hospitals, known as the district hospitals; and the state-level larger public hospitals are commonly known as the general hospitals. The differences between these two hospitals are based on the scope of medical services and scope of facilities that are being provided. At the district hospitals, clinical specialists from internal medicine, general surgery, and obstetrics and gynaecology are usually residents or visiting on a regular basis, whereas in the general hospitals, a more complete spectrum of secondary or tertiary medical/surgical care and services is available. In contrast with the private sector, patients in public healthcare services only have to pay nominal fees to obtain comprehensive primary and secondary healthcare services. ${ }^{10}$

Practising holistic medicine is not impossible, but often demands the FMSs to be aware of the comprehensive healthcare needs of a patient and to coordinate care for the patients and sometimes even for their family members. FMSs are therefore often involved in communication with the hospital specialists for patients who need secondary or tertiary care. Despite its 20-year history, neither the general public nor healthcare professionals understand the roles of family medicine and its practices. Realising the importance of other public healthcare professionals' expectation of the roles and functions of FMSs for the primary care services to be effective, the leaders of Family Medicine Specialists Association (FMSA), some universities and the Family Health Division (BPKK) and the Ministry of Health together initiated this study to examine the expectations of public healthcare providers/professionals (PHCPs) who are working closely with FMSs at the public health clinics. The results reported in this study were part of a larger cross-sectional study using postal survey throughout Malaysia. The objectives of the main study were to examine the perception of the PHCPs on the FMSs' (1) clinical competence and adherence to evidence-based medicine, (2) ethical practices, (3) safety issue in clinical practice, (4) professional attitude and teamwork and (5) involvement in research collaboration, scientific writing and publication. This study reports on the qualitative analyses of the expectations written by the PHCPs on FMSs in addition to the questionnaire-based items.

\section{METHODS}

\section{Setting}

We invited general hospitals and one district hospital from each state. The district hospital chosen was the one with which FMSs have close working relationships, and this decision was made after discussion with the state FMSs' representative/head. Using Excel's random number generator, we randomly selected five health clinics from every state based on the 2010 directory of health clinics with resident FMS. In the states with fewer than five clinics, all these clinics were included. We invited every state's health offices. District health offices that had health clinic/clinics selected within their districts were also invited.

\section{Subjects}

PHCPs at each of the participating health facilities were invited to participate voluntarily through a local site 
coordinator. Having previous personal encounters with FMSs was emphasised in the information sheet. 'Encounter' was defined as contacts through referral letters, direct consultation in-person or via telephone, emails and meetings either official or scientific. Only doctors, except house officers, with emphasis on the clinical specialist and consultants, were invited to the hospitals. All categories of PHCPs at the health clinics and health offices were invited except house officers, health attendants and those who had had psychiatric disorders that might impair judgement and memory.

\section{Sample size and sampling}

Sample size calculation was carried out using Epi Info 3.5.1. The estimated number of PHCPs from 25 public hospitals (14 general hospitals and 11 district hospitals), 67 public health clinics and 66 health offices was 5000 people. We expected poor perception of FMSs at $10 \%$ with a worst scenario of 5\%; the sample size was 228 with $99 \%$ CI. After accounting for response rate at $50 \%$ and $30 \%$ of incomplete questionnaires, the needed sample size became 1140 . With this estimated sample size, we decided that each state general hospital was to receive 50 questionnaires, each district hospital 20, each health clinic 10 and each health office 10 . In a few district hospitals with fewer than 20 specialists, we included medical officers with preferences to those who were more senior and/or had been working in the facility for a considerable number of years.

Sampling comprised initial invitation to all the selected public healthcare facilities and was followed by convenient sampling of participants at the participating centres. The initial invitation document package was sent to the heads of the healthcare facilities consisting of an endorsement letter from the director of the BPKK, the letter of MREC approval, a letter from the principal investigator of this study, the information sheet to the site coordinators with a study flow chart and a reply form. We requested personal particulars and contact details for the site coordinators and set a final date in the reply form. The selected centres were to fax the accomplished reply form to the principal investigator to indicate their willingness to participate. FMSs at the health clinics were avoided throughout the sampling process. Following the return of their reply form, the questionnaire sets were posted by courier service directed to the site coordinator at the participating centre. Site coordinators distributed the questionnaires according to the set criteria via convenient sampling. Completed questionnaires were returned to the investigators by courier service.

\section{The instrument}

The item assessing the respondent's expectation is worded as 'My expectation of a FMS is...'. This was one of the two open-ended items within the bigger study assessing the general perception of the respondents on the FMSs. The items within the main questionnaire were developed in English; it was then back-to-back translated into Malay. The questionnaire was tested for face and content validity with 10 PHCPs from each healthcare facility, and it was further improved based on their feedback. The English version was used for hospital specialists, whereas the Malay version was used for PHCPs at health clinics and health offices. We included a copy of the questionnaire in the other language (English to health clinics and health offices; Malay to hospitals) to all the participating centres to serve as a cross-reference for healthcare professionals who may be more proficient in the other language.

\section{Statistical analyses}

The outcome measures analysed in this study were the themes that emerged from the narrative analysis of PHCPs' written comments. ${ }^{12}{ }^{13}$ We removed any identifying data to ensure anonymity.

First, the written comments were read for understanding and transcribed into a Microsoft Word file. Difficult words were interpreted and discerned within the context of the comments. Only a few words were illegible but were transcribed into the Word file as written. These typed comments were then sent to the other investigators. After an initial review of 200 comments, four investigators, as a working group, first coded the text of the comments and then grouped these into subthemes, using an iterative process of discussion, refining and revision of the coding; a schema of subthemes was developed by consensus of all the members in the working group. Next, each of us then independently reviewed about 400 comments. At least two of us independently read and coded each other's 400 comments. Each subtheme was coded only once in each comment. New subthemes were added in the process when the existing subthemes were found inadequate for new comments. At the end of the individual coding process, we met in-group and derived consensus for each of the comments within the subthemes. With these subthemes, we then further conceptualised them into themes.

\section{RESULTS}

The response rate from the centres following the initial invitation was $40 \%(60 / 158)$. The participants' response rate was $58 \%(780 / 1345)$. We received an almost equal proportion of completed questionnaires from each category of the public healthcare facility, namely health clinics, health offices and hospitals (table 1). Four states (Melaka, Sabah, Pahang and Johor) contributed almost half $(47.6 \%)$ of the total responses, whereas Selangor, Putrajaya/Kuala Lumpur federal territories and Negeri Sembilan combined contributed only about $10 \%$ of the total responses.

Comments varied in length from 1 to 98 words. We identified a total of 21 subthemes through our coding process (table 2). The number of subthemes that we identified for each comment ranged from one to five. 
Table 1 Number and percentage of commented subthemes by the public healthcare providers/professionals from three healthcare facilities according to the six themes of expectation, $n(\%)$

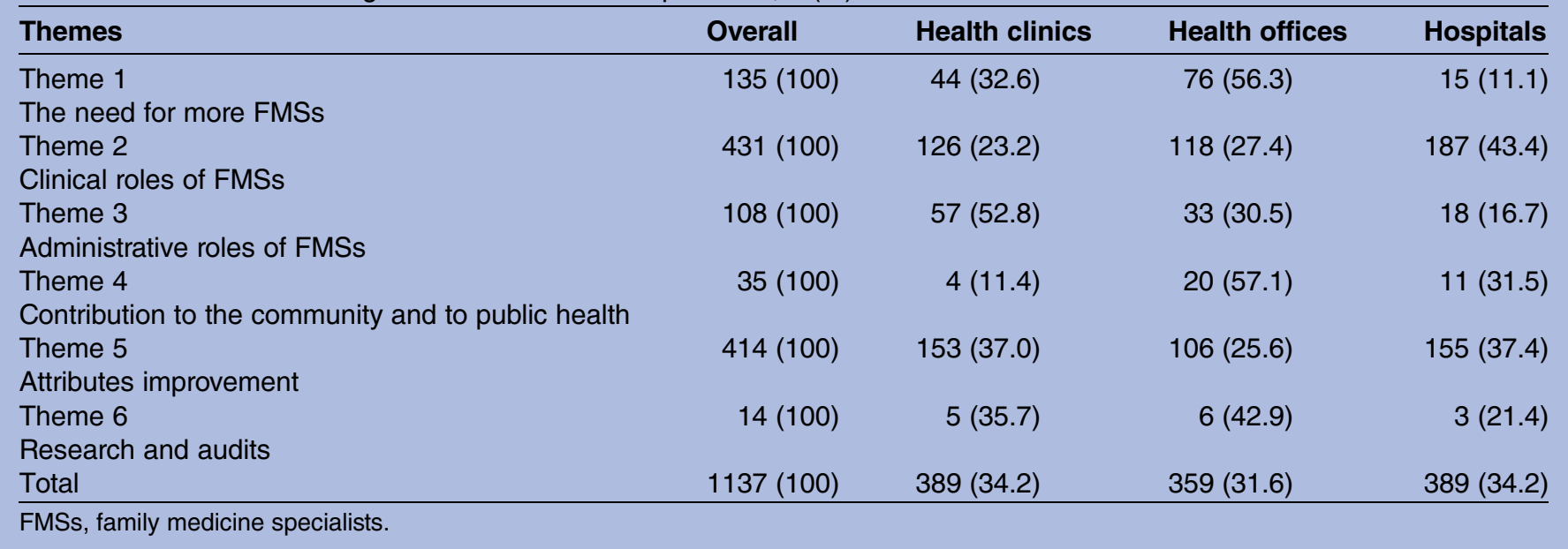

The six emerging themes are (1) the need for more FMSs, (2) clinical roles and functions of FMSs, (3) administrative roles of FMSs, (4) contribution to the community and to public health, (5) attributes improvement and (6) research and audits (table 2). While looking for relationships between the subthemes, we noticed diversity in the level of abstraction and aggregation of subthemes within themes. At the same time, we identified an underlying construct that allowed us to connect subthemes from different themes and to the different healthcare facilities.

In the overall comments, among the most common themes were 'clinical roles and functions of FMS', 'attributes improvement' and 'the need for more FMSs' (table 1). Unsurprisingly, hospitals' PHCPs had the highest expectation on FMS's clinical roles and functions, while clinics' PHCPs expressed the most expectation on FMSs' administrative roles (57/108 subthemes; table 1). Similarly, PHCPs at the health offices expressed the need for more FMSs (76/135 subthemes) and expected them to contribute more to the community and to public health programmes (20/35 subthemes; table 1$)$.

\section{Clinical roles of FMS}

We noticed that there were different expectations of FMSs from the three different categories of PHCPs. A majority of the PHCPs from hospitals expected FMSs to focus on clinical duties such as providing opinions to supervising doctors and staff at clinics, leading to better decisions and improved management of patients before

\section{Table 2 Expectation themes and subthemes}

\begin{tabular}{ll}
\hline Theme & Subtheme \\
\hline Need for more FMSs & 1. More FMSs at the district level \\
& 2. More FMSs at health clinics \\
3. More FMSs to visit other health clinics & 4. FMSs to stay longer at health clinics \\
Clinical roles and functions of FMSs & 5. Consultation by/supervision of MOs and staff \\
& 6. Better referral/FMSs to be consulted before referral \\
7. Training to staff-new update/case discussion/CME
\end{tabular}


referral to hospitals. The following quotations reflect the expectations of FMSs on their clinical duties as aforementioned.

MH36: Should be in clinic most of the working hours so that junior doctors can consult them...we have a lot of junior MO (medical officers) who just finished their housemenship,... and they are lacking in knowledge and experience...

JH08: Able to refer cases appropriately. Improve decision making for their medical officers. Training of all MOs at KK. High risk cases should be under their (FMS) follow up.

CH62: FMS is very important to improve or maintain good healthcare service, make appropriate referral and avoid unnecessary referral... They should always guide the new junior MOs that are being posted under their care.

MH18: (1) To identify care and refer to tertiary centre or general hospital. (2) To continue further management for patient from clinic, e.g. diabetes mellitus (DM)/ hypertension (HPT)/ischaemic heart disease and cancer treatments. (3) To stabilize patient's problems, e.g. DM/ HPT-optimize medication so that patient can proceed to surgery.

SH38: (1) Work up patient completely where appropriate before referral to specific discipline. (2) Communicate well with other discipline specialists to improve patient's health management...

PHCPs at the health clinics wished FMSs to have an effective appointment system and thus be able to see more patients at their clinics.

BC35: Need to concentrate on clinical than administrative duties. Do not get involved too much in the activities outside the clinic...

MC56: Need to concentrate on clinical duties and research work at the clinic level. Only need to attend meetings and courses which are important.

FMSs were expected to provide teaching and training to update their staff on current medical development, to promote teamwork within the clinic and with hospital staff.

DA08: To provide more continuous medical education $(C M E)$ activities at the clinic level for medical officers and paramedics-for knowledge update.

KH59: To train their medical officers in health clinics to be competent in patients' care and follow up.

BC43: Need to pay attention to those MOs who need guidance to solve their clinical case problems in the clinic.

SC23: (1) Works as a team. (2) To share information and knowledge in patient management.
JA25: FMSs need to have good relationship with their staffs and community; this will create a friendly working environment and provide effective services.

TA29: To have regular discussion with staffs.

\section{Attributes improvement}

The next most common expectation was on FMSs to continuously improve their personal and professional attributes. These unanimous expectations by all the PHCPs from the three healthcare facilities were that FMSs need to consolidate their medical knowledge and skills and to keep acquiring new medical updates.

AH61: FMS should be able to keep themselves up-to-date with the latest clinical practice guidelines and practice evidence-based care.

RH31: Their knowledge needs to be broad but sufficient in managing patients who are will be multidiscipline. Need to strengthen in (health) education and preventive medicine.

WH43: Able to treat and manage broad group of population and disease and also able to perform simple examination like ultrasound.

QC16: My hope in the FMS is that they need to be friendly, caring, humble and wise. They need to have good communication skills in speaking to staffs and patients, wise in everything, including well-informed on the latest drugs.

SC64: I hope FMSs can carry out their duties professionally and be patience in his duties. Need to show respect to staffs and patients.

\section{Needs for more FMIS}

This expectation came mainly from PHCPs at the health offices and clinics. The expressed need for more FMSs included having at least one at each health clinic and even more than one at the larger clinics. Many PHCPs at health clinics requested FMS not to be replaced too often or too soon. This expectation might indicate that shortages of FMSs had resulted in a high turnover rate or that FMSs' presence and services were appreciated by these PHCPs.

AA45: I hope each health clinic will have a FMS in future.

JH15: There should be a minimum of one FMS in each clinic either in urban or suburban area.

AA50: To increase the numbers of FMS (especially in the health clinics with heavy workload).

BC39: It is hoped that the FMSs will continue to stay at every health clinics and the numbers should be increased in order to improve the quality of care to patients. 
QA24: If possible, all clinics should have a FMS regardless of whether the clinic is an urban or a rural clinic.

CH10: ...the above impressions/expectations will remain idyllic only if the ongoing shortage of FMSs persists (it is not realistic if 1 FMS has to cover 5-6 different clinics in the district/state).

CH13: Need to increase the number of FMS/KK as they are also involved in other issues (meetings/conferences, etc) and might not be available at all times.

\section{Administrative roles}

In addition to the above expectations on FMSs to focus on clinical duty, there were some longings for FMSs to be involved in managing staff issues and in running the clinics. However, there were more direct and clear expectations on FMSs not to be involved in administrative duties at the health clinics and instead to focus on their clinical duties. Nevertheless, some PHCPs recognised that FMSs, being the only clinical specialists at health clinics, could not disassociate themselves from staff issues and practice management.

CC78: FMSs need to know the field of administration.

PA15: Need to have concern for his staffs and to show confidence in them.

DA03: To be more committed in fighting for his staffs' welfare.

MH51: Being a good manager in primary care team.

DC34: To monitor often the quality of treatment delivered by his staffs.

JH12: Overall manager of KK...

JH02: ...to lead government clinic (outside hospital) to a better governed and managed clinic.

\section{Involvement in the community and in public health}

As a clinical discipline that is community based, family medicine is indeed a unique specialty positioned between public health and hospital care. Hence, it was not surprising that many PHCPs at the health clinics and offices expected FMSs to be involved in community health programmes and investigative fieldwork during an outbreak as well as in managing public health issues.

QA31: To get involved directly in Millennium Development Goal 4 and 5 (MDG 4 and MDG 5) of the Family Health unit.

RA10: To get involved more in community activities such as delivering health education with the health promotion team.
JA31: I hope FMSs can be more available to solve public health problems in the field and not only be confined at the clinic.

RA06: To be more involved in public health programmes and not just as an expert for referral.

CA14: I hope good FMSs can...have administrative power over the district health officer...

\section{Research and audits}

This theme had very few comments. The low expectation on FMSs to conduct researches and to initiate audit activities could be due to the relatively more important expectation in the other areas of clinical practice.

JH06: To be involved in clinical research, monitor the operations of the health clinics, staff management so that screening protocols for different conditions are adhered to.

MC44: To conduct more research-based teaching, meeting, and discussion. More clinical audit sessions and provide maternal and child health once a week.

QA56: FMS should take the lead in improving the delivery of public healthcare system at clinic level, by auditing the clinical practices of other MOs and have a part in marking the annual job achievement (SKTs) of the MOs...

Few comments had reflected that some participants had concerns about the limitation of FMSs' administrative power in carrying out their responsibilities. Some PHCPs wanted FMSs to be recognised to have the same status as the hospital specialists. This domain had the least comments and almost all came from the nonhospital PHCPs.

SC40: I hope provision can be made available to the FMS to perform appropriate procedures so that patients do not need to go to the hospital for the procedures.

CA14: I hope good FMSs can be promoted....

JA18: The foreigner's fees to see a FMS should be similar to a hospital specialist.

\section{DISCUSSION}

This study reported on the qualitative analyses of respondents' written comments on expectations on FMSs: part of a larger national study on the perception of the Malaysian public healthcare professionals on FMSs (PERMFAMS). In spite of more written comments by the hospitals' PHCPs, the numbers of subthemes coded were about similar in the PHCPs' comments from the three different health facilities. As the coding strategy decided to provide quantitative weightage to the subthemes of expectation, such results indicated that non-hospital-based PHCPs had given broader 
expectation in a more concise manner compared with the hospital specialists who had elaborated their expectation mainly on clinical performance of FMSs. As this was a study on expectations on FMSs, it was not surprising that most expectations were directed towards improvement in clinical roles and functions, and the attributes of FMSs.

Among the different categories for the clinical roles and functions, we noticed that there were different expectations of FMSs from the three different categories of PHCPs. PHCPs at the health clinics wished FMSs to focus on clinical duties and be available for patients at health clinics. The availability of FMSs had become an issue in some of the clinics when the FMSs were also involved in administrative jobs that often required them to attend meetings. Family physicians' availability was an ongoing challenge and expectation elsewhere. ${ }^{14}$ Family physicians functioning within the Canadian long-term home services team could be disrupted due to their unavailability, irregularity or unpredictable presence. ${ }^{14}$ Thus, the presence and function of primary care physicians are increasingly being recognised within an effective healthcare system especially through their gate-keeper role to the more expensive hospital care.

PHCPs at the health clinics expected FMSs to organise more teaching and training sessions to update them on current medical knowledge. This aspiration was rightly expressed in view of FMSs often being the only resident clinical specialists at the health clinic level. However, meeting this expectation would often pose the challenge of finding suitable time for the staff without interrupting the operations of the clinic or taking out staff on weekends. In addition, with the busy clinic schedule of the FMSs, organising well-structured training with clear learning objectives for the different categories of clinic staff might be too demanding. Nevertheless, an active learner would be more likely to be an enthusiastic teacher. ${ }^{15}$ Hence, looking outwards and having an external conversation with other FMSs may encourage mutual learning and teaching opportunities for the FMSs and their staff. $^{16}$

Health offices' PHCPs expected FMSs to be more active team members and to take up leadership roles to foster stronger teamwork relationship among the multidisciplinary members. This observation by the closest health partners at the primary care level and immediate managing health offices of the FMSs and their clinics ${ }^{10}$ would be an invaluable feedback. Effective multidisciplinary teamwork, leading to an integrated approach of primary medical care delivery, is a crucial element for success. In this set-up, GPs were almost always considered to be the leaders. ${ }^{17}$ Good leadership would bring focused effort, clear direction, regular organisational adjustment and meetings that would maintain the cohesiveness of the health clinics. ${ }^{4}$

As expected, the hospital specialists generally expected the FMSs to supervise their staff performance, especially the medical officers, when providing treatments and referring patients to the hospitals. This expectation followed from the hospitals' specialist experience in receiving inadequate prehospital care and posthospital follow-up management of the patients by the medical officers. Thus, FMSs were highly expected by hospital specialists to improve management of patients, in their own respective disciplines, before referring to them as well as to continue posthospital care leading to reduced workload at the hospital specialist clinics. These are indeed great expectations and challenges for the family medicine specialty. To achieve this, FMSs are required to have a wide knowledge base in medicine and to keep these many pairs of knowledge-shoes shining. ${ }^{18-21}$ Quite a number of these expectations included more consultation and better communication between FMSs and the hospital specialists. Referrals that meet expectations could be learnt and would be achieved faster through more bidirectional dialogue and mutual respect plus understanding between FMSs and the hospital specialists. $^{22}{ }^{23}$ Collaborative care between these two sectors has its inherent difficulties and needs more than a formal encounter. ${ }^{24}$

The next major PHCPs' expectation was of FMSs to self-improve. FMSs were unanimously expected by all the PHCPs from the three healthcare facilities to consolidate their medical knowledge and skills and to keep acquiring new medical updates. Keeping up-to-date in knowledge and expertise is important, ever more so in engaging healthcare interactions with educated patients and in supporting their self-management behaviours and skills. ${ }^{25}$ Knowledge and skills improvements are almost always related to better care of patients at the clinic level. $^{2627}$ Providing continuous medical education to other staff would be an important part of task delegation and successful collaborative care for patients with a complex disease profile such as chronic diseases and multiple comorbidities. ${ }^{16} 28$ These educations were rightly expected for better quality medical care to be delivered by the FMSs and PHCPs, and a more active information transfer methods were preferred than a passive ones. ${ }^{29}$ In addition to this, PHCPs at health clinics expressed an almost equal number of expectations for the FMSs to improve on their personality, attitudes and behaviour in managing clinic issues and when dealing with staff. These included communication skills that showed mutual respect towards the staff, to listen and understand matters at hand before judging them. In English general practices whose staff reported a better team climate and team effectiveness using the team climate inventory (assesses perceptions of staff members of how people work together, how frequently they interact, whether teams have identified aims and objectives, and how much practical support and assistance are given towards new and improved ways of doing things). It was shown that quality of care, patient satisfaction, continuity of care and access to care were better. ${ }^{30}$ Physicians who had good interpersonal behaviour such as empathy and communication skills did improve 
patient satisfaction and adherence to therapy. ${ }^{31}$ For these attributes to be translated into effective teamwork, it would require all team members to practise these behaviours, not just the FMSs. ${ }^{17}$ These roles and responsibilities were also expected of the Canadian family physicians in running a cohesive and effective geriatric healthcare team. ${ }^{14}$

With the prevalent positive perception on FMSs, it was expected that many did express an expectation to have more FMSs at the primary medical care. The higher expectation came from PHCPs at the health offices and clinics, wishing that there was at least an FMS at every health clinic and even to have more than one FMS at the bigger or busier health clinics. However, the more realistic expectation for the near future is to have more FMSs at the district level to allow more visits to be made to other clinics without resident FMSs. Some PHCPs hoped that their current FMSs were to stay longer at the current posts indicating that FMSs turning-over or reposting to other clinics might be too fast to be desired.

There were more direct and clear expectations of FMSs to not get involved in administrative duties at the health clinics so as to be able to focus on clinical duties. However, some expectations did reveal that FMSs, being the only medical specialists at the health clinics, could not disassociate themselves from staff issues and practice management. FMSs in this country and family physicians worldwide are in fact trained in managing a health clinic. $^{32}$ As a clinical discipline that is community based, family medicine is indeed a unique specialty positioned between public health and hospital care. Therefore, it was not surprising that many PHCPs at the health clinics and offices expected FMSs to get involved in administrative works and community health programmes, to investigative fieldwork during an outbreak and even to manage public health issues. Thus, this study showed that there were still misconceptions on this specialty's clinical functions, and confusion on roles between the FMS and the public health physician.

Of the many expectations reported, the priority areas for FMSs to improve on are their clinical competency, and personal and professional attributes. ${ }^{21}$ FMSs do need to pay attention to medical updates, be cognisant about latest evidence and practise effective medical care in congruence to the clinical practice guidelines. ${ }^{33}$ This is then followed by being available more often at the clinic for the patients and clinic staff, by having an efficient appointment system, ${ }^{34} 35$ and by applying evidencebased medicine in managing or referring patients by themselves or through their medical officers. ${ }^{2}{ }^{36-38}$ Having an appropriate length of consultation time with each patient would help in a better clinical assessment of patients, decision-making and adherence to guidelines. ${ }^{39}$ Becoming an efficient clinician only is apparently insufficient and FMSs need to show a more caring attitude when dealing with clinic staff, and to always be thoughtful of their professional conduct when acting as a leader or as a team member. ${ }^{17}$ This, in turn, would create a good team climate leading to better chronic diseases management, preventive care services, patient satisfaction, continuity of care and access to specialist care. ${ }^{30}$

At higher levels, FMSA could organise relevant continuous professional development programmes on a regular basis consisting of scientific conferences, seminars or forums bringing together hospital specialists or experts to further understand essential updates in primary care medicine. ${ }^{40}$ Being the current main caretaker of this specialty in the public service, BPKK could work closely with the FMSs to improvise primary medical care. The Ministry of Health, through BPKK and state and district health offices, could do more by increasing FMS posts, allocating more resources that are in proportion to the workloads at each health clinic, as well as offering more opportunity for further training and promotion of the FMSs. Universities or training colleges that provide family medicine residency programmes should look into a more effective curriculum that could deliver the crucial clinical knowledge and incorporate desired skills in future FMSs. ${ }^{41}$ To be able to produce such FMSs, universities or training colleges need to have more capable academic staff, dedicated clinical site supervisors for curriculum implementation and regular formative and valid summative assessments.

\section{Strengths and limitations}

The strengths of this study were the nationwide scale of coverage with an almost equal participation of PHCPs from the three different public healthcare facilities. As the number of respondents decreased at state level, and with much unequal contribution from the three different healthcare facilities, the representativeness and generalisability of the findings to any of states are cautioned. We would suggest state-level or district-level studies, or even healthcare facility-level audits, conducted by personnel other than FMSs, to verify the results of this study. Recognising the sensitivity of the study on the FMSs and their potential influence at the health clinics, we adopted the non-inclusion of FMSs in the data collection process and also emphasised the same in the site coordinator information sheet. Despite these measures, we noticed that there were two health clinics whereby the FMSs had taken up the site coordinator's roles. Nevertheless, the responses from these clinics were not excessively favourable towards the FMSs; instead, they were more negative in one of the health clinics and rather average in the other. Thus, it appeared that there had not been any influence of the FMSs on the responses. Furthermore, with voluntary participation and confidential responses assured in this study, we can regard the comments to be of high honesty and sincerity and thus believable.

The other limitation would be that all investigators (except 1) were FMSs working in universities. Realising this to be a possible bias, we limited the number of FMS investigators when conducting the analyses. Of the four 
investigators, three were FMSs academicians and only one was a public FMS. During the report writing, all the involved investigators kept reminding each other to always be objective. However, this study served well the purpose of quantifying and confirming the general trends of certain perceptions of the PHCPs from the different public healthcare facilities on FMSs. Future works can further verify and explore the actual meanings of the reported expectations of FMSs among the health clinics' PHCPs and the relevance, practicality and prioritisation of certain clinical roles and functions as perceived by the hospitals' PHCPs. We recognise that it is also important to have more work that will reflect patients' expectations of the care provided to them and of the current healthcare system where they receive their regular healthcare from the FMSs to take patients' perspective into the care improvement exercise.

\section{CONCLUSION}

This study reported on the analysis of the written expectations of FMSs by PHCPs from three different public healthcare facilities. FMSs were expected to give more attention to their clinical duties. Executing this responsibility with competence included having up-to-date medical knowledge, better supervision of staff and medical officers under their care, fostering effective teamwork, and making appropriate referral. FMSs were also expected to behave professionally and respectfully in dealing with patients, and with staff when managing clinic issues concerning them. FMSs were expected to communicate more often with hospital specialists in patient care and referrals. The expectation for more FMSs was definite and strong, in contrast to low expectations of FMSs to be involved in research. Some mal-expectations on FMSs getting involved in community and public health programmes still exist. A better understanding of the family medicine specialty by PHCPs is therefore important for a truer expectation. This would avoid misunderstandings and an incorrect conclusion of FMSs being incompetent in the management of patients with undifferentiated symptoms and uncertain medical conditions that require unique skills in family medicine. Therefore, more initiatives are needed to assist PHCPs to have more cognisance of public family medicine practice that would lead to better healthcare delivery nationwide.

\author{
Author affiliations \\ ${ }^{1}$ Department of Family Medicine, Faculty of Medicine and Health Science, \\ Universiti Putra Malaysia, Serdang, Selangor, Malaysia \\ ${ }^{2}$ Health Clinic Seremban 2, Ministry of Health, Seremban, Negeri Sembilan, \\ Malaysia \\ ${ }^{3}$ Department of Family Medicine, Faculty of Medicine, University Kebangsaan \\ Malaysia, Cheras, Kuala Lumpur, Malaysia \\ ${ }^{4}$ Primary Care Medicine Discipline, Faculty of Medicine, Universiti Teknologi \\ MARA, Sungai Buloh, Selangor, Malaysia \\ ${ }^{5}$ Health Clinic Tampin, Ministry of Health, Tampin, Negeri Sembilan, Malaysia
}

Acknowledgements The authors would like to acknowledge the Director General of Health Malaysia for his permission to publish this report. The authors would like to record an appreciation to Dr Noridah Mohd-Salleh and
Dr Baizury Bashah for their contribution to the conception of the study design, research preparation stage and facilitation at the level of the Ministry of Health, Malaysia. They express their utmost gratitude to all the site coordinators who committed their time for the distribution and collection of the questionnaires. The authors are also grateful to the office staff at the Department of Family Medicine, Faculty of Medicine and Health Sciences, UPM who helped in the paper work of the study. Finally, they thank all the participants for their responses, without which this study would not have been possible.

Contributors B-HC and A-TC contributed to the acquisition of data. B-HC, $\mathrm{A}-\mathrm{TC}, \mathrm{MI}$ and $\mathrm{ZH}$ contributed to statistical analysis. B-HC drafted the manuscript. All authors contributed to the conception and design of the study. All authors contributed to the critical revision of the manuscript.

Funding This study received its funding from the Family Medicine Specialist Association Malaysia and a matching grant from the Universiti Putra Malaysia.

Competing interests None.

Patient consent Obtained.

Ethics approval This study has been approved by the Medical Research Ethics Committee (MREC), Ministry of Health Malaysia in December 2008. The Protocol Number from the National Medical Research Register is NMRR ID: 08-12-1167.

Provenance and peer review Not commissioned; externally peer reviewed.

Data sharing statement No additional data are available. There is another descriptive report on the sociodemographic factors and an overall perception based on itemised questionnaires, and a qualitative report on the impression of public healthcare professionals on family medicine specialists from this national survey. Both these manuscripts have been submitted to journals for consideration of publication.

Open Access This is an Open Access article distributed in accordance with the Creative Commons Attribution Non Commercial (CC BY-NC 3.0) license, which permits others to distribute, remix, adapt, build upon this work noncommercially, and license their derivative works on different terms, provided the original work is properly cited and the use is non-commercial. See: http:// creativecommons.org/licenses/by-nc/3.0/

\section{REFERENCES}

1. Martin JC, Avant RF, Bowman MA, et al. The Future of Family Medicine: a collaborative project of the family medicine community. Ann Fam Med 2004;2(Suppl 1):S3-32.

2. Frost DW, Toubassi D, Detsky AS. Rethinking the consultation process: optimizing collaboration between primary care physicians and specialists. Can Fam Physician 2012;58:825-8.

3. Cook RI, Render M, Woods DD. Gaps in the continuity of care and progress on patient safety. BMJ 2000;320:791-4.

4. D'Amour D, Ferrada-Videla M, San Martin Rodriguez L, et al. The conceptual basis for interprofessional collaboration: core concepts and theoretical frameworks. J Interprof Care 2005;19 (Suppl 1):116-31.

5. Committee On Quality Of Health Care In America. Crossing the quality chasm: a new health system for the 21st century. Washington, DC: Institute Of Medicine, 2001.

6. Adams K, Corrigan JM, eds. Priority areas for national action: transforming health care quality. Washington, DC: Institute of Medicine, 2003.

7. Pinder RJ, Greaves FE, Aylin PP, et al. Staff perceptions of quality of care: an observational study of the NHS Staff Survey in hospitals in England. BMJ Qual Saf 2013;22:563-70.

8. Guldberg TL, Lauritzen T, Kristensen JK, et al. The effect of feedback to general practitioners on quality of care for people with type 2 diabetes. A systematic review of the literature. BMC Fam Pract 2009;10:30.

9. Stevenson K, Baker R, Farooqi A, et al. Features of primary health care teams associated with successful quality improvement of diabetes care: a qualitative study. Fam Pract 2001;18:21-6.

10. Jaafar S, Mohd Noh K, Abdul Muttalib K, et al. Malaysia health system review. Health Syst Transition 2013;3:15-80. Asia Pacific Observatory on Health Systems and Policies. II. World Health Organization Regional Office for the Western Pacific. 
11. Yasin S, Chan CK, Reidpath DD, et al. Contextualizing chronicity: a perspective from Malaysia. Global Health 2012;8:4.

12. Corbin J, Strauss A. Grounded theory research: procedures, canons, and evaluative criteria. Qual Sociol 1990;13:3-21.

13. Patton MQ. Qualitative research \& evaluation methods. 3rd edn. Thousand Oaks, CA: SAGE Publications, Inc, 2001.

14. Wright $\mathrm{B}$, Lockyer J, Fidler $\mathrm{H}$, et al. Roles and responsibilities of family physicians on geriatric health care teams: health care team members' perspectives. Can Fam Physician 2007:53:1954-5.

15. Hartley S, Macfarlane F, Gantley M, et al. Influence on general practitioners of teaching undergraduates: qualitative study of London general practitioner teachers. BMJ 1999;319:1168-71.

16. Crabtree BF, Miller WL, McDaniel RR, et al. A survivor's guide for primary care physicians: building office relationships and interacting with the "local landscape" are the keys to resiliency. Here's how to do both. J Fam Pract 2009;58:E1.

17. Xyrichis $\mathrm{A}$, Lowton $\mathrm{K}$. What fosters or prevents interprofessional teamworking in primary and community care? A literature review. Int J Nurs Stud 2008;45:140-53.

18. Omar M, Tong SF, Saleh N, et al. A comparison of morbidity patterns in public and private primary care clinics in Malaysia. Malays Fam Physician 2011;6:19-25.

19. Chew BH, Than TL, Chew KS, et al. A one month review of the types of medical emergencies and their treatment outcomes at two urban public health clinics. Med J Malaysia 2012;67:571-6.

20. Kamarudin MF, Noh KM, Jaafar S. Morbidity profiles at three primary care clinics in Perlis, Malaysia. Med J Malaysia 2012;67:363-8.

21. Rourke J. The ideal family physician: W. Victor Johnston oration to the College of Family Physicians of Canada, Convocation, Winnipeg, Man, October 2007. Can Fam Physician 2008;54:18-21.

22. Thorsen O, Hartveit M, Baerheim A. General practitioners' reflections on referring: an asymmetric or non-dialogical process? Scand J Prim Health Care 2012;30:241-6.

23. Hoedeman R. Consultation letters for medically unexplained physical symptoms in primary care. Cochrane Database Syst Rev 2010;8(12):CD006524.

24. Legault $F$, Humbert J, Amos $S$, et al. Difficulties encountered in collaborative care: logistics trumps desire. J Am Board Fam Med 2012;25:168-76.

25. Snow R, Humphrey C, Sandall J. What happens when patients know more than their doctors? Experiences of health interactions after diabetes patient education: a qualitative patient-led study. BMJ Open 2013;3:e003583.

26. Ram P, Grol R, van den Hombergh $P$, et al. Structure and process: the relationship between practice management and actual clinical performance in general practice. Fam Pract 1998:15:354-62.
27. Wong ST, Watson DE, Young E, et al. What do people think is important about primary healthcare? Health Policy 2008;3: 89-104.

28. Archer J, Bower P, Gilbody S, et al. Collaborative care for depression and anxiety problems. Cochrane Database Syst Rev 2012;10:CD006525.

29. Hulscher ME, Wensing M, van Der Weijden $\mathrm{T}$, et al. Interventions to implement prevention in primary care. Cochrane Database Syst Rev 2001;1:CD000362.

30. Campbell SM, Hann M, Hacker J, et al. Identifying predictors of high quality care in English general practice: observational study. BMJ 2001;323:784-7.

31. Kim SS, Kaplowitz S, Johnston MV. The effects of physician empathy on patient satisfaction and compliance. Eval Health Prof 2004;27:237-51.

32. Rivo ML. Practicing in the new millennium: do you have what it takes? Fam Pract Manag 2000;7:35-40.

33. Bagley B. The new model of family medicine: what's in it for you. Fam Pract Manag 2005;12:59-63.

34. The College of Family Physicians of Canada. Best advice-timely access to appointments in family practice. http://webcache. googleusercontent.com/search?q=cache:GKhxtoSI_TMJ:toolkit.cfpc. ca/en/files/2012\%2520Final\%2520Best\%2520Advice\% 2520Enhancing\%2520Timely\%2520Access\%2520.pdf $+\& \mathrm{~cd}=9 \& \mathrm{hl}=\mathrm{en} \& \mathrm{ct}=\mathrm{clnk \& gl}=\mathrm{my}$.

35. Mitchell V. Same-day booking: success in a Canadian family practice. Can Fam Physician 2008:54:379-83.

36. Faulkner A, Mills N, Bainton D, et al. A systematic review of the effect of primary care-based service innovations on quality and patterns of referral to specialist secondary care. $\mathrm{Br} \mathrm{J}$ Gen Pract 2003:53:878-84.

37. Vachon B, Desorcy B, Camirand M, et al. Engaging primary care practitioners in quality improvement: making explicit the program theory of an interprofessional education intervention. BMC Health Serv Res 2013;13:106.

38. Akbari A, Mayhew A, Al-Alawi MA, et al. Interventions to improve outpatient referrals from primary care to secondary care. Cochrane Database Syst Rev 2008;4:CD005471.

39. Tsiga $\mathrm{E}$, Panagopoulou $\mathrm{E}$, Sevdalis $\mathrm{N}$, et al. The influence of time pressure on adherence to guidelines in primary care: an experimental study. BMJ Open 2013;3:e002700.

40. Dwamena F, Holmes-Rovner M, Gaulden CM, et al. Interventions for providers to promote a patient-centred approach in clinical consultations. Cochrane Database Syst Rev 2012;12:CD003267.

41. Soklaridis S, Oandasan I, Kimpton S. Family health teams: can health professionals learn to work together? Can Fam Physician 2007:53:1198-9. 